\title{
SOME OBSERVATIONS ON THE PERMANGANATE TEST.
}

By A. Dupre, Рн.D., F.R.S., \&c.

Communicated to the Society of Public Analysts on 14th December, 1881.

THE Committee of the Society having put forward a certain method for estimating the amount of oxygen absorbed, it may perhaps be advisable to give some reasons for that method to show that it is really an advantage compared with the older one.

The first point for consideration is, Does permanganate suffer decomposition spon. taneously at 80 degrees Fahr. in the presence of so much sulphuric acid? I have made a great many experiments, and find that, in really pure water, there is, practically, no decomposition at 80 degrees Fahr. after four hours; or at any rate the amount decomposed is always well within the limits of experimental exror. It may, therefore, be taken as established that if the experiment is carried out in a closed vessel there is no spontaneous decomposition, and consequently all the oxygen that is found to be absorbed at that temperature must have been absorbed by some substance present in the water. In an open vessel this could not be assumed, as I do not think it would be possible to heat water in 
an open vessel to 80 degrees with permanganate without some decomposition. The point is of importance, because it does away with the second, blank experiment so strongly recommended by Tidy. He makes two experiments; he adds the same amount of permanganate to the water being examined and to distilled water. He takes the distilled water as his standard, and at the end of three hours calculates from that the oxygen absorbed in the other. If the vessel is closed this is entirely unnecessary, and one great source of error, the possibility of traces of impurity present in the distilled water or absorbed from the air, is removed.

This closed vessel makes, however, another precaution necessary. I do not know whether all our analysts do so, but it is necessary to standardize the permanganate in a closed vessel, for if you standardize in a beaker less hyposulphite is required, unless you do the titration very rapidly. If you do it slowly you always get a lower result in the beaker than in the bottle. I should not be astonished if some of the results of no oxygen absorbed are really due to the loss occasioned in that way.

In some of my experiments the amount of oxygen absorbed, determined as rapidly as possible in a bottle, or in a beaker, was found to be 11.2 to 11.25 e.c. of hypo. Taking ten minutes for the titration, the hypo. comes down to 10.9 , or sometimes even lower in the beaker, but remains the same in the bottle.

I must say I was rather astonished at that. I expected to find more iodine liberated by giving a little time, but instead of that there is less, and there is no doubt that a small amount of iodine volatilizes during the titration. If it is done in a bottle, and left even for half an hour, identical results are obtained. A result I did not expect. I expected a considerable increase in iodine liberation by the action of the sulphuric acid on the iodine.

The next point is with regard to the variation of the oxygen absorbed by the variation of temperature.

Here $I$ found that if the water is very pure the amount of oxygen absorbed does not vary much with the temperature. The variation remains within the limits of experimental error, wherever it is done at 32 degrees or 80 degrees. If we take a somewhat less pure water there is, however, a very perceptible difference, and the more impure the water the greater is the difference in the amount of oxygen absorbed according to temperature. In the Trafalgar Square water the variation is only between $\cdot 017$ and 03 . It seems proportionally large, but it is quite within the limits of error. It represents less than half a tenth of permanganate, which I may fairly take to be within experimental error. The same result comes out even if you increase the temperature up to 100 degrees.

Taking the water supplied to the Westminster Hospital, that absorbed 0526 at 54 degrees, and $\cdot 065$ at 80 degrees, which is of course a very measurable increase. If we take a still more impure water the difference becomes greater. A water I had from Farnborough gave 0.0395 at 32 degrees, 0.0554 at 60 degrees, and 0.0613 at 80 degrees, this being a very measurable increase.

It follows that if we have a water of great purity it really matters very little how we keep the temperature; but if our water is only of moderate purity, it is a matter of very great importance indeed to keep the temperature fairly uniform.

If the water is still more impure the difference increases, and 1 degree or 2 degrees Fahr. might then make a sensible difference.

It would be desirable to have some experiments made at lower temperatures, and I should not be surprised if a comparison between the action at a low and high temperature 
would enable us to make distinctions between waters which the higher temperature alone does not enable us to do. We might distinguish between two kinds of organic matter, which, at a higher temperature, give the same result, but a different result at a lower temperature.

Variation in Trms.--In a pure water the variation in time is not great-the oxidation comes to an end sooner than in an impure water; so that no great difference is found, whether the water is treated for three or four hours. Dr. Tidy told me that he never had a water that absorbed after three hours. That is so with pure waters, but not with impure ones, as in these there is a very decided increase from the three to four hours.

The Farnborough water, for example, in three hours absorbed $\cdot 055$, and at four hours .061 grains of oxygen, a very noticeable increase; and $I$ have found the same in other waters. I have no doubt that with a really impure water four hours is not enough to give to the limit of what the permanganate process will do.

The next point to which I directed my attention is the variation of oxidation by varying the amount of permanganate; and here, also, the result corresponds very much with the preceding. With a fairly pure water it matters nothing whether you add 10 or 20 c.c. If you work well you come to the same result.

In the Trafalgar Square water, with 10 c.c. of permanganate I got 0.009 , and with 20 c.c. $0 \cdot 010$, which is practically identical. Taking the Chelsea water, to which a little blood had been added, with 10 c.c. of permanganate, the oxygen absorbed was 0.115 ; but with 20 e.c. it had increased to $0 \cdot 124$.

I next tried phosphoric acid in place of sulphuric acid, bearing in mind that the latter decomposes the hydro-iodic acid, and renders the titration thereby somewhat inaccurate, and more particularly that it interferes with the sharpness of the final reaction.

Taking phosphoric acid (in equivalent proportions) the water remains generally more clear, and the final reaction is beautifully sharp. It has also a slight effect in making the oxygen absorbed, with varying amounts of permanganate, somewhat more uniform.

It diminishes, however, very appreciably the amount of oxygen absorbed. It has thus the advantage of rendering the end reaction sharper, and, perhaps, of making the amount of oxygen absorbed somewhat more independent of the amount of permanganate taken; but it diminishes the oxygen absorbed very considerably, and I have, therefore, returned to the use of sulphuric acid.

I next made a great many experiments with the permanganate test, with a view to throw some light on the organic matter present.

The first thing I did was to boil the water, and I found, to my great astonishment, that it increased the oxygen absorbed. That is to say, taking our Chelsea Company's water and estimating the oxygen atsorbed direct, then boiling it for an hour, and again estimating the oxygen absorbed, an increase is observable.

As we always heat our water for some time with sulphuric acid, I thought I would try boiling the water with sulphuric acid. I found, on the whole, a noticeable increase in the amount of oxygen absorbed. Only occasionally did I find the reverse, and that was always when there was a considerable amount of nitrates present. With that exception I always found an increase, which seemed to be the greater the impurer the water, or perhaps the more recent the contamination. Or to put it broadly, if the organic matter is 
already altered by natural action in the water, it does not get altered by boiling with sulphuric acid, a point which, if it should be confirmed by other observers, is of considerable importance.

I will now give you a few waters that have been purposely polluted. By far the most striking results were obtained by pure crystal sugar, a remarkably pure sample of which I obtained from Mr. Wigner. Adding sugar to the Trafalgar Square water increased the oxygen absorbed in four hours but slightly; but if the water is boiled with sulphuric acid it goes up to tenfold that amount.

Oxygen Absorbed in Four Hours at 80 deg. F.

\begin{tabular}{|c|c|c|c|c|c|c|c|c|c|}
\hline By & fal & qua & ater & & & & & $0 \cdot 010$ & rain: \\
\hline , & $n$ & ", & ," & +1 grail & sugar & & .. & $0 \cdot 017$ & ," \\
\hline ", & $"$ & , & $"$ & $+1=$ & $" 1$ & boiled $1^{\text {th }}$ with & 10 c.c, acid & $0 \cdot 160$ & 3 \\
\hline " & ", & " & , & $+0 \cdot 1$, & ," & 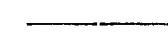 & $-\ldots$ & 0.009 & \\
\hline , & " & $"$ & $"$ & +0.1, &,$" 1$ & boiled $1^{\mathrm{h}}$ with & 10 c.c. acia & 0.016 & \\
\hline
\end{tabular}

This result led me to expect that the acid might be the means of distinguishing between vegetable and animal matters, but so far I have not been able to do so. I also tried alkali, and I had some hopes by its means of being able to distinguish between the two kinds of matter; but, though I took the utmost care to get the alkali pure, yet I found the purest soda, after acidifying with sulphuric acid, absorbed a considerable amount of permanganate. I suppose that the pure sulphate of soda has a sensible effect on permanganate. At any rate, however pure the water was, I never could get it to give no oxygen absorbed when ever I boiled with alkali ; while boiling with sulphuric acid did not affect pure water.

Gelatink.--Taking 2.27 grains of gelatine per gall., I found that the water absorbed in four hours 036 without boiling, and after boiling $\cdot 039$ - a very slight increase indeed. In half-an-hour it absorbed 017 without boiling, and $\cdot 036$ after boiling. The effect is thus very slight in four hours, but very perceptible in half-an-hour.

Urine.-30 grains added per gall., and treated it in the same way.

Oxygen Absorbed per Gali. IN-

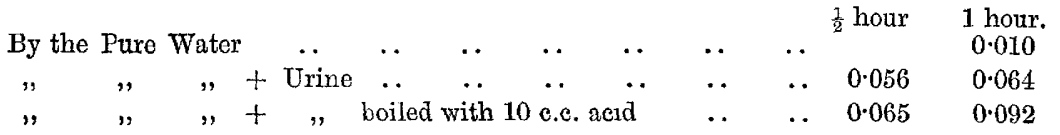

The difference is much larger than any experimental error would be.

EGg Albumen.- The amount I added was 1 grain to the 2 litre, which comes to 0.035 of dried albumen per gall. I have only the four-hour result, which is $\cdot 014$ without boiling, 0.016 after boiling. The pure water, as before, absorbed 0.01 .

The last substance tried was starch, which was rather disappointing. I expected to get a very noticeable increase. I added 2.27 grains per gall., and the oxygen absorbed without boiling was 008 (in fact, exactly the same as the pure Trafalgar water), after boiling $0 \cdot 020$. The starch was taken, boiled up into a thin mucilage and then added to the water.

No doubt the proportional increase is considerable, yet the actual increase, compared to the quantity taken, is nothing like what one would expect. Evidently this boiling for an hour with 10 c.c. acid per $\frac{1}{4}$-litre does not convert the starch into sugar.

In reply to questions, Dr. Dupré said : I have made it a point to test all waters for nitrous acid with starch and iodide, but I use sometimes Gries' test, which is more 
delicate. With these tests you may get the most intense colouration, and yet not the slightest discolouration of permanganate. All the experiments were made with soda made from sodium. I dissolved that to the corresponding strength of our sulphuric acid, and added permanganate and put it overnight in my warm chamber. I added permanganate till it was distinctly green, and yet when $10^{\prime \prime}$ of this soda solution were added to Trafalgar Square water, together with 20 c.c. sulphuric acid, there was always a noticeable decomposition of permanganate.

One of my reasons for bringing this subject forward in an incomplete form is that I may induce some of our younger members to devote some of their time to such experiments as these, my engagements not allowing me to carry the experiments further at the present time. 